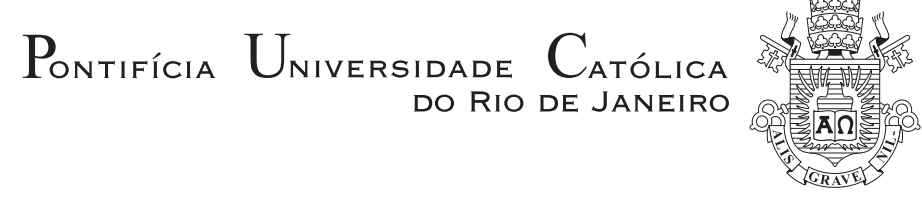

Guilherme Augusto Ferreira Lima

\title{
Eliminando Redundâncias no Perfil NCL EDTV
}

\section{Dissertação de Mestrado}

Dissertação apresentada ao programa de Pós-graduação em Informática da PUC-Rio como requisito parcial para obtenção do título de Mestre em Informática.

Orientador: Prof. Luiz Fernando Gomes Soares 


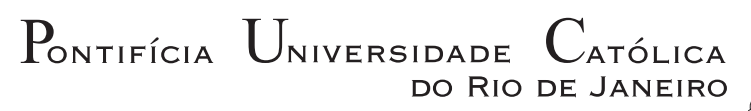
DO RIO DE JANEIRO

\title{
Guilherme Augusto Ferreira Lima
}

\section{Eliminando Redundâncias no Perfil NCL EDTV}

\begin{abstract}
Dissertação apresentada como requisito parcial para obtenção do grau de Mestre pelo Programa de Pósgraduação em Informática da PUC-Rio. Aprovada pela Comissão Examinadora abaixo assinada.
\end{abstract}

Prof. Luiz Fernando Gomes Soares

Orientador

Departamento de Informática - PUC-Rio

Prof. Renato Fontoura de Gusmão Cerqueira

Departamento de Informática - PUC-Rio

Prof. Marcelo Ferreira Moreno

Universidade Federal de Juiz de Fora - UFJF

Prof. José Eugenio Leal Coordenador Setorial do Centro Técnico Científico - PUC-Rio

Rio de Janeiro, 13 de abril de 2011 
Todos os direitos reservados. É proibida a reprodução total ou parcial do trabalho sem autorização da universidade, do autor e do orientador.

\section{Guilherme Augusto Ferreira Lima}

Graduou-se em Sistemas de Informação pela PUC-Rio em 2008. Atualmente integra o grupo de pesquisadores do Laboratório Telemídia, onde desenvolve pesquisas na área de sistemas hipermídia e televisão digital.

Ficha Catalográfica

Lima, Guilherme Augusto Ferreira

Eliminando Redundâncias no Perfil NCL EDTV / GuiIherme Augusto Ferreira Lima; orientador: Luiz Fernando Gomes Soares. - Rio de Janeiro: PUC-Rio, Departamento de Informática, 2011.

v., 70 f.: il. ; $29,7 \mathrm{~cm}$

1. Dissertação (mestrado) - Pontifícia Universidade Católica do Rio de Janeiro, Departamento de Informática.

Inclui referências bibliográficas.

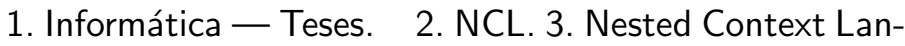
guage. 4. NCL EDTV profile. 5. NCL Raw profile. 6. Eliminação de redundâncias. 7. Conversão de documentos. I. Soares, Luiz Fernando Gomes (Luiz Fernando Gomes Soares). II. Pontifícia Universidade Católica do Rio de Janeiro. Departamento de Informática. III. Título. 
Aos meus pais pela confiança. A Bianca pelo carinho e paciência. 


\section{Agradecimentos}

Agradeço ao meu orientador Prof. Luiz Fernando Gomes Soares pelo exemplo, atenção aos detalhes e sabedoria; e ao Prof. Edward Hermann Haeusler pelo apoio. Agradeço também a todos os colegas do Lab. TeleMídia. Em especial, a Marcelo Moreno, Márcio Moreno, Romualdo Costa, Carlos Salles, Rafael Savignon, Francisco Sant'Anna, Carlos Batista, Roberto Azevedo, José Geraldo de Souza e Eduardo Araújo, pelas críticas e sugestões. E, ao restante da equipe, Álvaro Veiga, Bruno Lima, Felipe Nogueira, Felipe Nagato, Ricardo Rios, Luciana Redlich, Vinicius Lago pelo apoio. Finalmente, agradeço ao Conselho Nacional de Desenvolvimento Científico e Tecnológico (CNPq) pelo suporte financeiro sem o qual este trabalho não seria possível. 


\section{Resumo}

Lima, Guilherme Augusto Ferreira; Soares, Luiz Fernando Gomes. Eliminando Redundâncias no Perfil NCL EDTV. Rio de Janeiro, 2011. 70p. Dissertação de Mestrado - Departamento de Informática, Pontifícia Universidade Católica do Rio de Janeiro.

A implementação de uma máquina de apresentação NCL, ou formatador, é uma tarefa complexa. Essa complexidade decorre, principalmente, da distância semântica que existe entre os documentos NCL, especificações declarativas de alto-nível, e as API que o formatador utiliza para apresentá-los, em geral imperativas e de baixo-nível. Quanto maior a distância, maior a complexidade do mapeamento e, conseqüentemente, da sua implementação que tende a ser ineficiente e não-confiável. Este trabalho apresenta um novo perfil para a linguagem NCL, chamado NCL Raw, que elimina as redundâncias do EDTV o principal perfil da NCL 3.0 - e, de certa forma, aproxima os documentos da máquina. O perfil Raw captura apenas os conceitos essenciais do EDTV que por sua vez podem ser usados para simular a linguagem completa. Ou seja, podemos usar o Raw como uma linguagem intermediária mais simples para a qual documentos EDTV podem ser convertidos antes de serem apresentados. Esta dissertação discute as possíveis arquiteturas para conversores NCL e apresenta uma implementação de um conversor de documentos (EDTV para Raw).

\section{Palavras-chave}

NCL, Nested Context Language, NCL EDTV profile, NCL Raw profile, eliminação de redundâncias, conversão de documentos. 


\section{Abstract}

Lima, Guilherme Augusto Ferreira; Soares, Luiz Fernando Gomes (Advisor). Eliminating Redundancies from NCL EDTV Profile. Rio de Janeiro, 2011. 70p. MSc. Dissertation - Departamento de Informática, Pontifícia Universidade Católica do Rio de Janeiro.

The implementation of a NCL presentation engine, or formatter, is a complex task. This complexity is mainly due to the semantic distance between NCL documents, high-level declarative specifications, and the API used by the formatter to present them, in most cases low-level and imperative. The greater the distance, the greater is the complexity of this mapping and, consequently, of its implementation, which is more likely to become inefficient and bug-prone. This work presents a new NCL profile, called NCL Raw, which eliminates most of the redundancies present in EDTV - the main profile of NCL 3.0 - and, in a certain way, reduces the distance between the documents and the machine. Raw profile captures only EDTV's essential concepts, which in turn can be used to simulate the whole language defined by EDTV itself. In other words, we can use the Raw profile as a simpler intermediate language to which EDTV documents can be converted before being presented. This dissertation discusses alternative architectures for NCL converters and presents the implementation of a document converter (from EDTV to Raw).

\section{Keywords}

NCL, Nested Context Language, NCL EDTV profile, NCL Raw profile, redundancies elimination, document conversion. 


\section{Sumário}

1 Introdução 13

1.1 Motivação . . . . . . . . . . . . . . . . . . . . 13

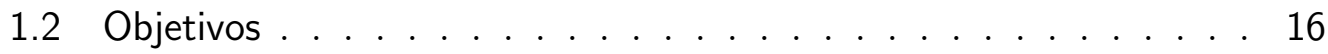

1.3 Organização da Dissertação . . . . . . . . . . . . . . . . . 17

2 O Perfil EDTV 18

2.1 Estrutura Básica . . . . . . . . . . . . . . . . . . . . . . . . 18

2.2 Elementos do Corpo . . . . . . . . . . . . . . . . . . . . . . . 19

2.2 .1 Nós de conteúdo . . . . . . . . . . . . . . . . . 20

2.2 .2 Nós de composição . . . . . . . . . . . . . . . . . . . . . . . . . 22

2.2 .3 Nós de ligação (elos) . . . . . . . . . . . . . . . . . . . . . 24

2.2 .4 Nó settings . . . . . . . . . . . . . . . . . . . . . . . 26

2.3 Elementos do Cabeçalho . . . . . . . . . . . . . . . . . . . . 27

2.3.1 Base de documentos importados . . . . . . . . . . . 27

2.3 .2 Regiões . . . . . . . . . . . . . . . . . . . 28

2.3 .3 Descritores . . . . . . . . . . . . . . . . . . . . . . 28

2.3 .4 Transições . . . . . . . . . . . . . . . . . . . 29

2.3 .5 Conectores . . . . . . . . . . . . . . . . . . . 30

2.3 .6 Regras . . . . . . . . . . . . . . . . 30

3 Eliminação das Redundâncias 32

3.1 Procedimentos de Eliminação . . . . . . . . . . . . . . . . . . . 32

3.1.1 Elementos <importNCL $>$ e $<$ importedDocumentBase $>$. . 32

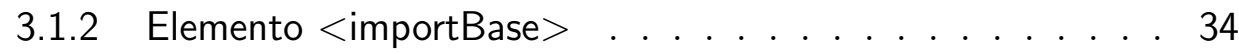

3.1.3 Elementos $<$ region $>$ e $<$ regionBase $>\ldots . . . . . . .35$

3.1.4 Elementos $<$ transition $>$ e $<$ transitionBase $>\ldots . . . . .36$

3.1.5 Elemento $<$ descriptor $>$. . . . . . . . . . . . . . . 37

3.1.6 Elementos $<$ descriptorSwitch $>$ e $<$ descriptorBase $>$. . . . 38

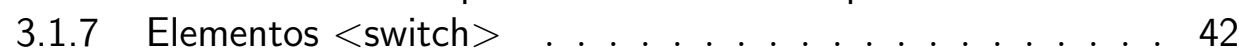

3.2 Perfil Raw . . . . . . . . . . . . . . . . . . 43

4 Conversor EDTV $\rightsquigarrow$ Raw $\quad 45$

4.1 Arquitetura . . . . . . . . . . . . . . . . . . . 45

4.1.1 Conversão de documentos . . . . . . . . . . . . . . . . 46

4.1.2 Conversão de comandos de edição . . . . . . . . . . . . . . 47

4.2 Implementação . . . . . . . . . . . . . . . . . . . . . . . 49

4.2 .1 Estrutura da Libncc . . . . . . . . . . . . . . . . . 50

4.2 .2 API da Libncc . . . . . . . . . . . . . . . . 51

5 Conclusão $\quad 55$

$\begin{array}{ll}\text { Referências Bibliográficas } & 56\end{array}$

A Gramática do Perfil EDTV 57 
B LibPlayer $\quad 63$

B.1 API de player . . . . . . . . . . . . . . . 66 63

B.2 Plugins . . . . . . . . . . . . . . . . . . 67

B.3 Loop de eventos . . . . . . . . . . . . . . . . . . . . . . 68

B.4 Dependências . . . . . . . . . . . . . . . . 69 


\section{Lista de Figuras}

1.1 Arquitetura básica do formatador NCL . . . . . . . . . . . . . . . . . 14

2.1 Estrutura básica de um documento EDTV . . . . . . . . . . . . . . . 19

2.2 Estrutura de um objeto de mídia EDTV . . . . . . . . . . . . 20

2.3 Estrutura de um contexto EDTV . . . . . . . . . . . . . . . 22

2.4 Estrutura de um switch EDTV . . . . . . . . . . . . . . . 23

2.5 Máquina de estados de evento . . . . . . . . . . . . . 25

2.6 Estrutura de um elo EDTV . . . . . . . . . . . . . . . . 26

2.7 Estrutura de uma base de documentos importados EDTV . . . . . . . 27

2.8 Estrutura de uma base de regiões EDTV . . . . . . . . . . . . . . 28

2.9 Estrutura de uma base de descritores EDTV . . . . . . . . . . . . . . 29

2.10 Estrutura de uma base de transições EDTV . . . . . . . . . . . . . . . 29

2.11 Estrutura de uma base de conectores EDTV . . . . . . . . . . . . . . 30

2.12 Estrutura de uma base de regras EDTV . . . . . . . . . . . . . . . . . 31

4.1 Processo de conversão de documentos . . . . . . . . . . . . . . . . . 46

4.2 Arquitetura do conversor de documentos . . . . . . . . . . . . . . . 47

4.3 Processo de conversão de comandos de edição . . . . . . . . . . . . . . 48

4.4 Pipeline de conversão da Libncc . . . . . . . . . . . . . . . . . . . . . 50

B.1 Exemplo de programa LibPlayer . . . . . . . . . . . . . . . . . . 66

B.2 Loop de eventos da LibPlayer . . . . . . . . . . . . . . . . . . . . . 68 


\section{Lista de Tabelas}

2.1 Algumas propriedades pré-definidas do EDTV . . . . . . . . . . . . . . 21

2.2 Atributos opcionais do elemento $<$ transition $>\ldots$. . . . . . . . . 26

2.3 Atributos opcionais do elemento $<$ transition $>\ldots$. . . . . . . . . . . 29

3.1 Gramática do perfil NCL Raw . . . . . . . . . . . . . . . . . . . 43

A.1 Gramática do perfil EDTV . . . . . . . . . . . . . . . . . . . . . 57

B.1 Propriedades reconhecidas por cada classe de player . . . . . . . . 65 
Everything should be made as simple as possible, but no simpler.

- Albert Einstein 